ISSN 1991-8631

Original Paper

http://indexmedicus.afro.who.int

\title{
Biosynthesis of lipophilic compounds in tomato fruit
}

\author{
Djédoux Maxime ANGAMAN ${ }^{1,2^{*}}$, Irene PATERAKI ${ }^{2}$, Montserrat BUSQUETS ${ }^{2}$ and \\ Albert BORONAT ${ }^{2}$ \\ ${ }^{1}$ Unité Pédagogique et de Recherche (UPR) en Biochimie et Microbiologie, Université Jean Lourougnon \\ Guédé 02 BP 150 Daloa 02, Côte d'Ivoire. \\ ${ }^{2}$ Departament de Bioquímicai Biologia Molecular, Facultat de Biologia, Universitat de Barcelona, Avda. \\ Diagonal 643, 08028-Barcelona, Spain. \\ *Corresponding author, E-mail: angamanmaxime@yahoo.fr; Tel: (+225) 40106635
}

\begin{abstract}
Metabolic engineering approaches to increasing lycopene contents in tomato fruit have always led to moderate increases of this metabolite. This reflects, at least in part, the lack of knowledge about the mechanisms underlying the regulation of carotenoid biosynthesis and accumulation as well as the rest of metabolic processes operating in the chromoplasts. A study performed with chromoplasts to know the origin of the precursors for carotenoids biosynthesis using a variety of ${ }^{14} \mathrm{C}$-labelled precursors showed that the most important incorporation was found in lipids. This study aims to understand the biochemical and metabolic processes operating during tomato fruit ripening. Labelling studies showed an efficient incorporation of ${ }^{14} \mathrm{C}$ acetate not only into fatty acids and sterols (as expected) but also into lycopene, suggesting that the cytosolic mevalonate pathway was also contributing to carotenoid biosynthesis during fruit ripening. This observation has been confirmed using ${ }^{14} \mathrm{C}$-mevalonate, which served as a precursor for the synthesis of sterols and carotenoids.
\end{abstract}

(C) 2013 International Formulae Group. All rights reserved.

Keywords: Carotenoid, sterol, labelling assay, pericarp, chromoplasts.

\section{INTRODUCTION}

Tomato is the main source for dietary lycopene in western diets since, in contrast to others carotenoids, the natural sources of this compound are limited (Fraser and Bramley, 2004). Thus, the lycopene content of tomato fruit is a relevant issue in the commercialization of fresh and processed tomato. The increase of lycopene content in tomato fruit represents a major target in tomato breeding programs.

Lycopene begins to accumulate at breaker stage of tomato fruit (when a red/orange coloration becomes apparent) and its concentration increases up to 500-fold in ripe fruits (Fraser et al., 1994). During this process, the transcription of the genes encoding phytoene synthase (PSY) and phytoene desaturase (PDS) is up-regulated, whereas the expression of the mRNAs of lycopene $\beta$-cyclase (LYC-B) and lycopene $\varepsilon$ cyclase (LCY-E) is strongly reduced (Hirschberg, 2001). The activation of carotenoid biosynthesis during tomato fruit ripening also correlates with the up-regulation of genes encoding regulatory enzymes of the 2-C-methyl-D-erythritol 4-phosphate (MEP) pathway, like 1-deoxy-D-xylulose 5-phoshate synthase (DXS) (Lois et al., 2000). 
The incorporation assays using isolated chromoplasts revealed that all the precursors tested were incorporated at low levels into carotenoids (Angaman et al., 2012). Since the same precursors were efficiently incorporated into lipids it was hypothesized that some unknown factors could be limiting carotenoid biosynthesis and/or accumulation in the isolated plastids. To confirm this hypothesis we performed similar incorporation assays using tomato fruit pericarp tissue.

\section{MATERIALS AND METHODS} Plant material

Tomato (Lycopersicon esculentum Mill. cv. Ailsa Craig) plants were grown under standard greenhouse conditions. Fruits were collected at different stage of development and after breaking. Fruits were processed immediately after harvesting or stored at $4{ }^{\circ} \mathrm{C}$ and used within the next 2-3 days in the case of chromoplast extraction.

\section{In vivo labelling using tomato fruit pericarp tissue samples}

In vivo labelling was performed using the method described by Enfissi et al. (2005) and modified in some details. Uniform pericarp tissue samples of about $0.25 \mathrm{~g}$ were cut using a scapel and incubated in $25 \mathrm{mM}$ Tris- $\mathrm{HCl} \mathrm{pH} 7.5$ buffer, containing $200 \mathrm{mM}$ sorbitol, $10 \mathrm{mM}$ sucrose, $50 \mathrm{mM} \mathrm{KCl,} 5 \mathrm{mM}$ succinic acid, $1 \mathrm{mM}$ DTT, $1 \mathrm{mM}$ EGTA, 5 $\mathrm{mM} \mathrm{MgCl}_{2}, 0.1 \mathrm{mM}$ thiamine, and $9.2510^{4}$ Bq $\left[2-{ }^{14} \mathrm{C}\right]$ pyruvate, $\left[2-{ }^{14} \mathrm{C}\right]$ acetate or $\left[2-{ }^{14} \mathrm{C}\right]$ mevalolactone (American Radiolabeled Chemicals, USA) as substrate, in a total volume of $500 \mu \mathrm{l}$. Pericarp samples were incubated at room temperature for $24 \mathrm{~h}$ with gentle shaking. After incubation, the labelling solution was removed and the tissue washed with $300 \mu$ l of water. Samples were frozen with liquid nitrogen and ground in a mortar.

\section{Isolation and purification of tomato chromoplasts}

Tomato fruit chromoplasts were isolated using the method described by Angaman et al. (2012). All materials and solutions were kept at $2-4{ }^{\circ} \mathrm{C}$ during the isolation and purification process. Fruits (about $300 \mathrm{~g}$ ) were washed with $2.5 \% \mathrm{NaCl}$ in distilled water for $15 \mathrm{~min}$. About $200 \mathrm{~g}$ of pericarp tissue, after the removal of the skin, seeds and the gelatinous material of the locular cavities, was cut into small pieces with a razor blade and mixed with 2 volumes of buffer A (100 mM Tris- $\mathrm{HCl}$ pH 8.2, $0.33 \mathrm{M}$ sorbitol, $2 \mathrm{mM} \mathrm{MgCl}, 10 \mathrm{mM} \mathrm{KCl}, 8 \mathrm{mM}$ EDTA, $10 \mathrm{mM}$ ascorbic acid, $5 \mathrm{mM} \mathrm{L-}$ cysteine, $1 \mathrm{mM}$ PMSF, 1\% PVPP and $1 \mathrm{mM}$ DTT). After homogenization with a Waring blender (three pulses at low speed) the homogenate was first filtered through 8 layers of gauze and then through 2 layers of Miracloth (Calbiochem). The debris retained in the gauze layers were recovered, mixed with one volume of buffer $A$ and homogenized again. After filtration through gauze and Miracloth layers as described above, the homogenates were mixed and centrifuged for $2 \mathrm{~min}$ at $200 \times \mathrm{g}$. The supernatant was recovered and centrifuged for $10 \mathrm{~min}$ at $5,000 \times \mathrm{g}$. The obtained pellet was resuspended in $50 \mathrm{ml}$ of buffer B (buffer A without PVPP) and centrifuged for $10 \mathrm{~min}$ at $5,000 \times \mathrm{g}$. The pellet resuspended in $4 \mathrm{ml}$ of buffer $\mathrm{B}$ and chromoplasts were fractionated by ultracentrifugation on a discontinuous sucrose gradient $(15 \%, 30 \%, 40 \%$ and $50 \%$ in Tris- $\mathrm{HCl} \mathrm{pH} 7.4$ supplemented with $1 \mathrm{mM}$ DTT) for $1 \mathrm{~h}$ at $100,000 \times \mathrm{g}($ Beckman SW 28 rotor). Chromoplast fractions banding at the $15-30 \%, 30-40 \%$ and $40-50 \%$ interfaces (Figure 1A) were recovered by gentle aspiration with a Pasteur pipette. The collected fractions were washed with one volume of buffer B and chromoplasts were recovered by centrifugation (10 min, $5,000 \times \mathrm{g})$.

\section{Uptake assays of radiolabelled precursors}

Uptake assays were performed using the double silicone oil layer centrifugation method described by Gross et al. (1990) and modified by Weber et al. (2000). Chromoplasts were resuspended in $100 \mu \mathrm{l}$ of incorporation buffer (100 mM HEPES-KOH pH 7.6, $0.33 \mathrm{M}$ sorbitol, $2 \mathrm{mM} \mathrm{MnCl}_{2}, 10 \mathrm{mM}$ $\mathrm{MgCl}_{2}, 1 \mathrm{mM}$ NADP, $1 \mathrm{mM}$ NADPH, $5 \mathrm{mM}$ ATP, $20 \mu \mathrm{M}$ FAD) containing 0.5, 1, 2 and 4 $\mathrm{mM}$ of cold substrate (malate, pyruvate or 
glucose) supplemented with 3.125 Bequerel $(\mathrm{Bq})$ of the corresponding labelled substrate $\left[1-{ }^{14} \mathrm{C}\right]-$ IPP and incubated at $25{ }^{\circ} \mathrm{C}$. At the end of the specified incubation periods, chromoplast samples were loaded on top of a density gradient containing (from bottom to top) $100 \mu \mathrm{l}$ of glycerol:methanol $(2: 1[\mathrm{v} / \mathrm{v}])$, $50 \mu \mathrm{l}$ of silicone oil AR200 (Fluka, Deisenhofen, Germany), $50 \mu \mathrm{l}$ of buffer B containing $220 \mathrm{mM}$ sorbitol and $110 \mathrm{mM}$ sucrose, $50 \mu \mathrm{l}$ of silicone oil AR200 in along polyethylene microcentrifuge tube of $0.4 \mathrm{ml}$ (500-Q Quality Scientific Plastics) and then centrifugated at $14,000 \times \mathrm{g}$ during $30 \mathrm{~s}$. Tubes were frozen in liquid nitrogen and the lower part containing the chromoplast pellet cut and placed into a vial containing $2 \mathrm{ml}$ of scintillation liquid (Ecoscint $\mathrm{H})$. The incorporated radioactivity was counted in a liquid scintillation analyzer (TRI-CARB 2100 TR, Packard).

\section{Analysis of radiolabelled compounds using TLC and autoradiography}

For $250 \mathrm{mg}$ of fruit pericarp samples or $100 \mu \mathrm{l}$ of chromoplast suspension samples, labelled lipophilic compounds were extracted using the following method: $1 \mathrm{ml}$ of hexane: acetone: methanol (2: 1: 1, v/v) mixture was added (the microcentrifuge tubes were protected from the light with aluminium foil) and shacked with a chain dump form 15 min. Distilled water $(100 \mu \mathrm{l})$ was then added and vigorously mixed using a vortex. After centrifugation for $3 \mathrm{~min}$ at $3,000 \times g$, the organic fraction was kept in a new eppendorf. The extraction process was repeated and the new organic was mixed with the previous one and dried under a current gas $\mathrm{N}_{2}$. Samples were dissolved in $100 \mu 1$ of chloroform and used for counting the radioactivity or analysis by TLC (SIL G-25 UV 254 plates).

Different mobile phases were used for the TLC analysis, hexane: toluene (7:3, v/v) for highly hydrophobic compounds, like carotenoids, and chloroform: methanol: water (65: 25: 4, v/v) for more or less hydrophobic compounds, like free sterol, steryl ester. After drying, the TLC plates were placed in contact with Fujifilm screens for bioimaging analyzer
BAS-IPMS 2325 and scanned with Bioimager (Bio-Rad).

\section{RESULTS \\ Labeling studies using $\left[2-{ }^{14} \mathrm{C}\right]$-pyruvate, $[2-$ $\left.{ }^{14} \mathrm{C}\right]$-acetate and $\left[{ }^{14} \mathrm{C}\right]$-mevalonate}

Since pyruvate was considered a common precursor for both carotenoid and lipid biosynthesis in the plasts, the first radiolabelled precursor tested was $\left[{ }^{14} \mathrm{C}\right]$ pyruvate. As expected, labelled pyruvate was incorporated into carotenoids at rates significantly higher. When TLC plates were developed with hexane:toluene $(7: 3, \mathrm{v} / \mathrm{v})$, lycopene and $\beta$-carotene were separated together with steryl ester, quinone and a very hydrophobic compound not yet identified (Figure 1A). Although some labeled compound could be easily identified like carotenoids the identification of the rest of the labeled compounds was undertaken using candidate reference compounds and gas chromatography - electron impact mass spectrometry (GC-EI).

The presence of labelled sterols and sterol derivatives were further confirmed using $\left[{ }^{14} \mathrm{C}\right]$-acetate. In pericarp tissue, exogenously supplied $\left[{ }^{14} \mathrm{C}\right]$-acetate should be first converted to $\left[{ }^{14} \mathrm{C}\right]$-acetyl-CoA to serve as a precursor for the synthesis of fatty acids (via the plastidial fatty acid synthase complex) and sterols (through the operation of the cytosolic MVA pathway). As expected, the results shown in Figures 1A and 2A revealed patterns of labeled compounds that were very similar to those obtained with $\left[{ }^{14} \mathrm{C}\right]$-pyruvate. Surprisingly, carotenoids and quinone were also identified among the labelled compounds. Since there are no reports on the conversion of acetate (or acetyl-CoA) into pyruvate or glyceroaldehyde 3-phosphate (GA3P) (the precursors of the plastidial MEP pathway), the most likely explanation for the labelling of carotenoids from $\left[{ }^{14} \mathrm{C}\right]$-acetate was the exchange of IPP derived from the MVA pathway between the cytosol and the chromoplasts.

To confirm the unexpected results indicating the possible contribution of the MVA pathway in the synthesis of plastidial isoprenoids during tomato fruit ripening, 
labelling experiments using $\left[{ }^{14} \mathrm{C}\right]$-mevalonate were performed. The obtained results showed an efficient incorporation of radioactivity into sterols (Figure 2), carotenoids (Figure 1C) and quinone (Figure 1C).

\section{Uptake of $\left[{ }^{14} \mathrm{C}\right]$ IPP into isolated tomato fruit chromoplasts}

To further confirm that the IPP used for carotenoid biosynthesis in the pericarp tissue samples was derived from the MVA pathway we studied the uptake of radiolabelled IPP into isolated tomato fruit chromoplasts. As shown in Figure 3A, IPP was incorporated into chromoplasts, yielding the highest incorporation rates at a concentration of 2 $\mathrm{mM}$.
To analyze the products labelled from IPP, chromoplasts were incubated with $\left[{ }^{14} \mathrm{C}\right]-$ IPP for $24 \mathrm{~h}$ and the radiolabelled products analyzed by TLC and autoradiography as previously described. The obtained results revealed that phytoene was the only carotenoid efficiently labelled (Figure 3B). Other plastidial isoprenoids, like plastoquinone, geranyl geranyl diphosphate (GGPP) were also identified among the labelled compounds.

All together these results reinforce the unexpected involvement of the MVA pathway in the synthesis of carotenoids during fruit ripening and thus the occurrence of an active cross-talk between the MVA and the MEP pathways in the tomato fruit.
A

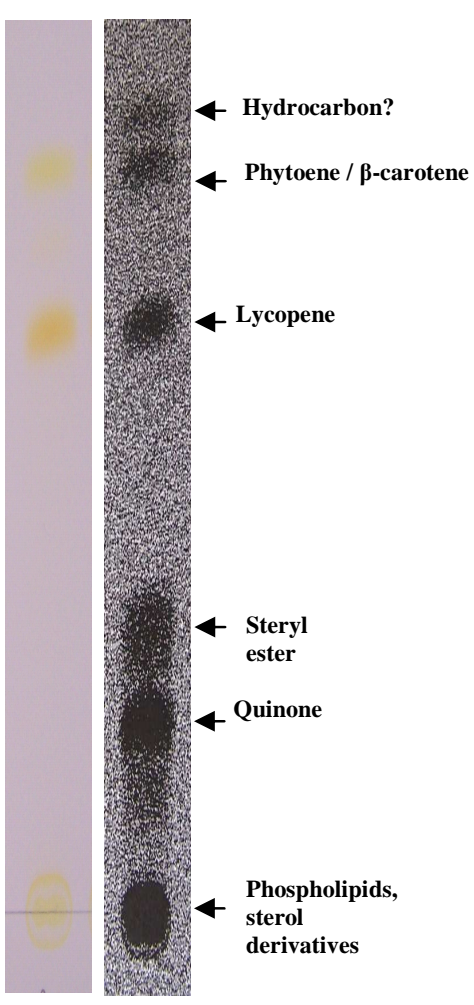

B

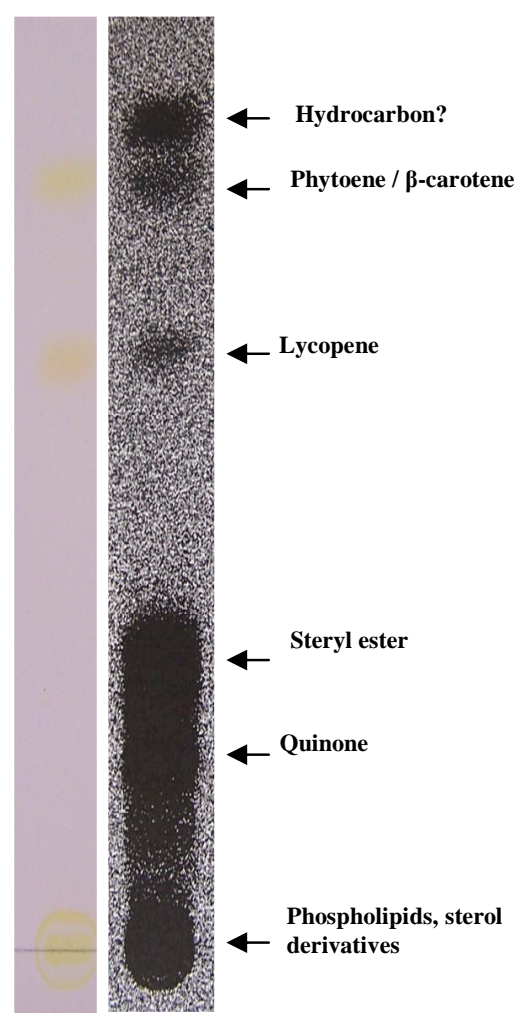

C

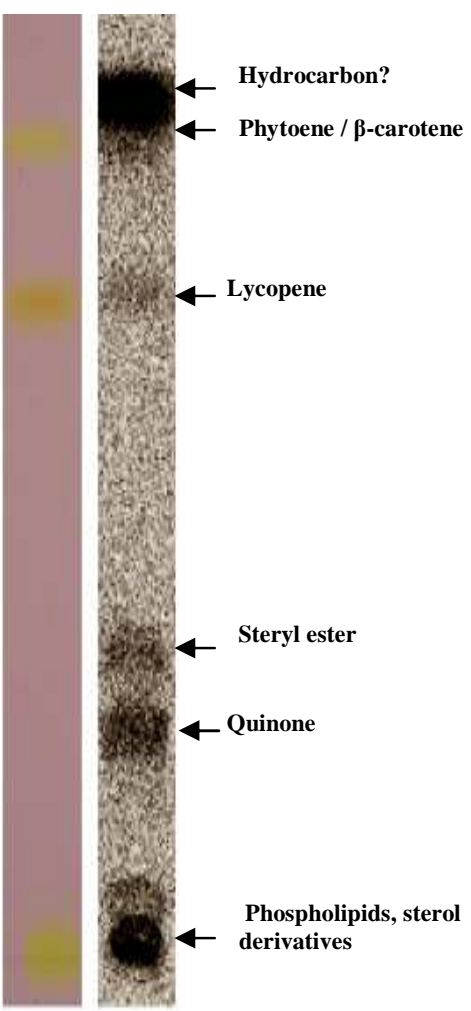

Figure 1: Analysis of radiolabelled compounds present in the organic fraction after extraction of pericarp tissue incubated with $\left[2-{ }^{14} \mathrm{C}\right]$-pyruvate $(\mathrm{A}),\left[2-{ }^{14} \mathrm{C}\right]$ acetate $(\mathrm{B}),\left[2-{ }^{14} \mathrm{C}\right]$ mevalonate $(\mathrm{C})$. TLC was developed with hexane: toluene $(7: 3, \mathrm{v} / \mathrm{v})$. 


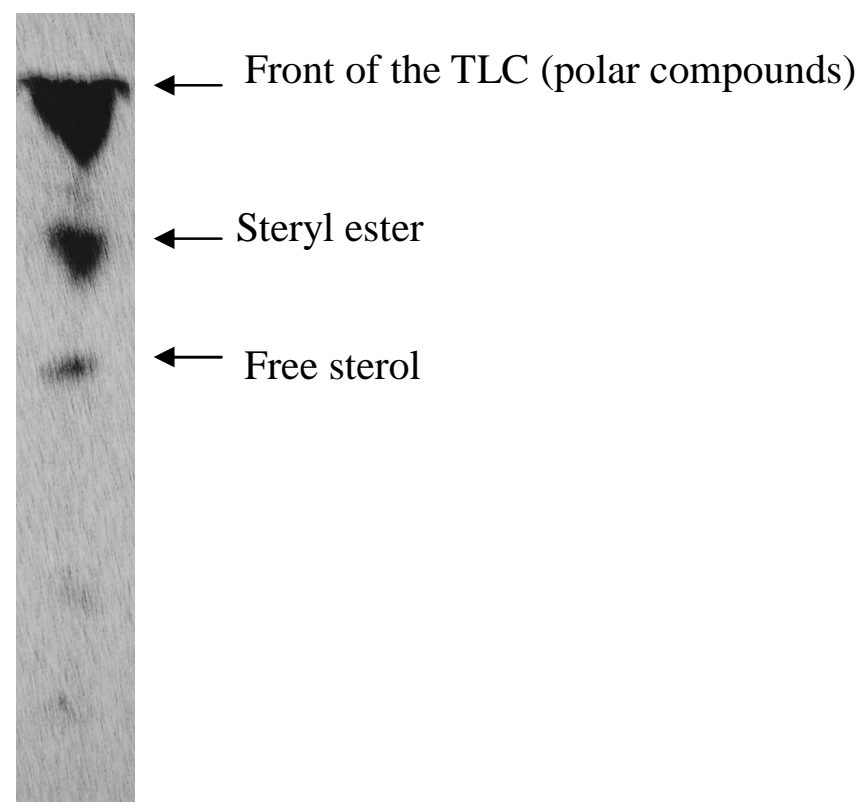

Figure 2: Analysis of radiolabelled compounds present in the organic fraction after extraction of pericarp tissue incubated with $\left[{ }^{14} \mathrm{C}\right]$ mevalonate. TLC plates were developed with chloroform: methanol: $\mathrm{H}_{2} \mathrm{O}$ (65: 25:4, v/v).

A

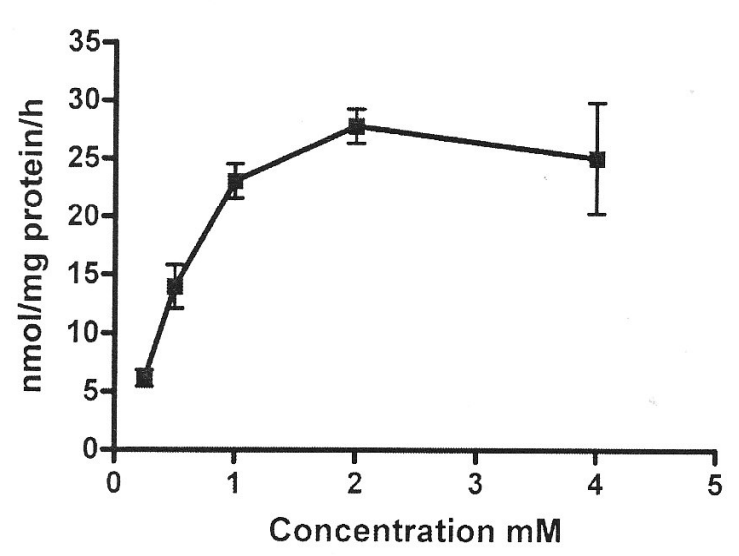

B

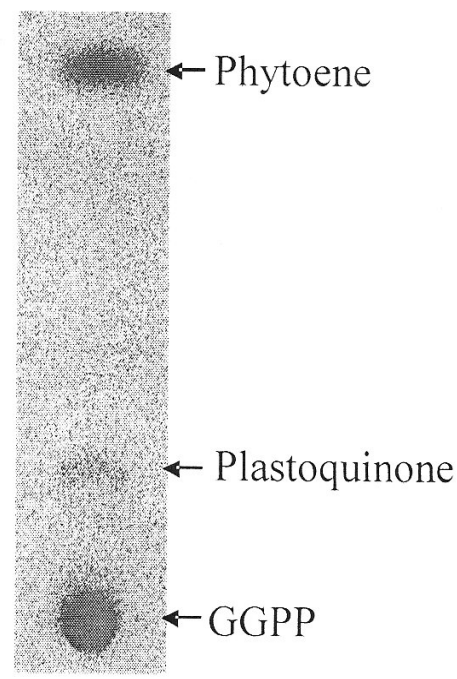

Figure 3: Uptake of ${ }^{14} \mathrm{C}$-IPP $(0.78 \mathrm{~Bq})$ into isolated tomato fruit chromoplasts. Incubation time was $60 \mathrm{~min}$ (A). Analysis of radiolabelled compounds present in the organic fraction after extraction of isolated chromoplasts incubated with $\left[{ }^{14} \mathrm{C}-\mathrm{IPP}\right]$ for $24 \mathrm{~h}$. Eluent for TLC was hexane: toluene (7:3, v/v) (B). 


\section{DISCUSSION}

Sterol biosynthesis during tomato fruit ripening

Incorporation studies in pericarp tissue samples using $\left[{ }^{14} \mathrm{C}\right]$-pyruvate have shown that sterols are also actively synthesized during tomato fruit ripening. In agreement with this, labelling experiments using $\left[{ }^{14} \mathrm{C}\right]$-acetate (precursor of $\left[{ }^{14} \mathrm{C}\right]$-acetyl-CoA) resulted in an efficient incorporation of radioactivity into lipids and sterols. The finding that sterols were synthesized de novo during tomato fruit ripening was totally unexpected considering that authoritative reviews on plant isoprenoid biosynthesis had never reported that sterol biosynthesis and, consequently, the operation of the MVA pathway could be an active process in ripening tomato fruit (Gillaspy et al., 1993; Weissenborn et al., 1995).

Sterols are important components of biological membranes that contribute to their fluidity and permeability (Hartmann, 1998). Furthermore, sterols are also the precursors of brassinosteroids, growth regulators involved in plant growth and development (Schaller et al. 2003). In this respect, it is worth noting that brassinosteroids have been reported to induce ripening of tomato fruit pericarp discs (Vardhini\&Rao, 2002), thus suggesting a role of this hormone in tomato fruit ripening. Recently the role of sterols (or their precursors) in plastid biogenesis has also been reported (Babiychuck et al., 2008).

The sterol composition of mature green tomato fruit is similar to that of tomato leaves, although quite unusual relative to most other plant species (Duperon et al., 1984). Acylated steryl glucosides are the most abundant sterols in the fruit. Together with steryl glucosides, they account for $85-90 \%$ of total sterols. During tomato fruit ripening, total sterol content increases and sterol composition and the profile of conjugated sterols are significantly modified (Chow and Jen, 1978;
Whitaker, 1988). The sterol content and profile of tomato fruits has been reported to change in response to chilling and heat treatment (Moreau, 2003). Because of the profound effects of sterols on the physical properties of biological membranes, it has been proposed that these changes may be involved in the regulation of membrane properties in response to environmental signals (Moreau, 2003). However, since sterols are also known to play a relevant role in plant growth and development, their involvement in the regulation of fruit ripening cannot be ruled out.

\section{The Mevalonate pathway contributes to carotenoid biosynthesis in tomato pericarp tissue}

Labelling experiments of pericarp tissue using $\left[{ }^{14} \mathrm{C}\right]$-acetate (a precursor of $\left[{ }^{14} \mathrm{C}\right]$-acetyl-CoA) resulted in an efficient incorporation of radioactivity into the lipid and the sterol fractions. Surprisingly, incorporation of radioactivity was also found in carotenoids. The unexpected labelling of carotenoids from $\left[{ }^{14} \mathrm{C}\right]$-acetate could only be explained assuming that IPP synthesized in the cytosol by the mevalonate (MVA) pathway was taken up by chromoplasts and then incorporated into carotenoids. This hypothesis has been confirmed after finding the incorporation of $\left[{ }^{14} \mathrm{C}\right]-\mathrm{MVA}$ into carotenoids in pericarp tissue samples and the uptake $\left[{ }^{14} \mathrm{C}\right]-\mathrm{IPP}$ into isolated chromoplasts. These results were indicative of a crosstalk between the MVA and the MEP pathway during tomato fruit ripening never reported before. Although is well documented that the operation of the MEP pathway is essential for carotenoid biosynthesis during the early stages of tomato fruit ripening, the crosstalk between the MVA and the MEP pathway in others stages of fruit ripening has never been explored. 
The crosstalk between the MVA and the MEP pathways observed in ripening tomato fruit could certainly open new possibilities in the development of new metabolic engineering strategies aimed at increasing carotenoid biosynthesis in tomato fruit. Thus, it could be proposed that an upregulation of the MVA pathway might have a positive effect in the content of fruit carotenoids by providing an additional supply of IPP to the chromoplasts. However, transgenic tomato plants constitutively overexpressing the Arabidopsis HMGR1 isoform have shown increased levels of sterols in the fruit without significant changes in carotenoid content (Enfissi et al., 2005). A possible explanation could be a limiting activity of the putative plastidic IPP translocator involved in the uptake of cytosolic IPP into the chromoplasts.

\section{ACKNOWLEDGEMENTS}

This work was supported by grants of the Spanish Ministerio de Ciencia e Innovación (BIO2009-09523 to Albert Boronat, including European Regional Development Funds), the Spanish ConsoliderIngenio 2010 Program (CSD2007-00036 Centre for Research in Agrigenomics) and the Generalitat de Catalunya (2009SGR0026). Djédoux Maxime Angaman was recipient of a predoctoral fellowship from Generalitat de Catalunya (DEBEQ).

\section{REFERENCES}

Angaman DM, Petrizzo R, Hernandez-Gras F, Romero-Segura C, Pateraki I, Busquets M, Boronat A. 2012. Precursor uptake assays and metabolic analyses in isolated tomato fruit chromoplasts. Plant Methods, 8(1): 1-10.

Babiychuk E, Bouvier-Navé P, Compagnon V, Suzuki M, Muranaka T, Van Montagu M, Kushnir S, Schaller H. 2008. Allelic mutant series reveal distinct functions for Arabidopsis cycloartenol synthase 1 in cell viability and plastid biogenesis. Proc. Natl. Acad. Sci. U S A., 105: 3163-3168.

Bugos RC, Chiang VL. 1995. RNA isolation from plant tissues recalcitrant to extraction in guanidine. Biotechniques, 19(5): 734-737.

Chow ETS, Jen JJ. 1978. Phytosterol biosynthesis in ripening tomatoes. $J$. Food. Sci., 43: 1424-1426.

Duperon R, Thiersault M, Duperon P. 1984. High level of glycosylated syterol species of Solanum and sterol changes during the development of tomato. Phytochemistry, 23: 743-746.

Enfissi EMA, Fraser PD, Lois ML, Boronat A, SchuchW, Bramley PM. 2005. Metabolic engineering of the mevalonate and non mevalonate isopentenyl diphosphate-forming pathways for the production of health-promoting isoprenoids in tomato. Plant Biotechnol. J., 3: 17-27.

Fraser PD, Truesdale MR, Bird CR, Schuch W, Bramley PM. 1994. Carotenoid biosynthesis during tomato fruit development (Evidence for tissue-specific gene expression). Plant Physiol., 105: 405-413.

Fraser PD, Bramley PM. 2004. The biosynthesis and nutritional uses of carotenoids. Prog. Lipid Res., 43: 228 265.

Gillaspy G, Ben-David H, Gruissem W. 1993. Fruits: a developmental perspective. Plant Cell, 5: 1439-1451.

Gross A, Brückner G, Heldt HW, Flügge U-I. 1990. Comparison of the kinetic properties, inhibition and labelling of the phosphate translocators from maize and spinach mesophyll chloroplasts. Planta, 180: 262-271. 
Hartmann MA. 1998. Plant sterols and membrane environment. Trends Plant Sci. 3: 170-175.

Hirschberg J. 2001. Carotenoid biosynthesis in flowering plants. Curr. Opin. Plant Biol., 4: 210-218.

Lois LM, Rodriguez-Concepcion M, Gallego F, Campos N, Boronat A. 2000. Carotenoid biosynthesis during tomato fruit development: regulatory role of 1deoxy-D-xylulose 5-phosphate synthase. Plant J., 22: 503-513.

Moreau RA, Whitaker BD, Hicks KB. 2002. Phytosterols, phytostanols, and their conjugates in foods: structural diversity, quantitative analysis, and healthpromoting uses. Prog. Lipid Res., 41: 457-500.
Schaller H. 2003. The role of sterols in plant growth and development. Prog. Lipid Res., 42: 163-175.

Vardhini BV, Rao SSR. 2002. Acceleration of ripening of tomato pericarp discs by brassinosteroids. Phytochemistry, 61: 843-847.

Weissenborn DL, Denbow CJ, Kaine M, Lang SS, Yang Z, Yu X, Cramer CL. 1995. HMG-CoA reductase and terpenoid phytoalexins: molecular specialization within a complex pathway. Physiol. Plant, 93: 393-400.

Whitaker BD. 1988. Changes in steryl lipids content and composition of tomato fruit during ripening. Phytochemistry, 27: 3411-3418. 